\title{
Cannabis and psychosis: what do we know and what should we do?
}

\author{
Marco Colizzi and Robin Murray
}

\section{Summary}

It is now incontrovertible that heavy use of cannabis increases the risk of psychosis. There is a dose-response relationship and high potency preparations and synthetic cannabinoids carry the greatest risk. It would be wise to await the outcome of the different models of legalisation that are being introduced in North America, before deciding whether or not to follow suit.

\section{Declaration of interest}

None.

\section{Copyright and usage}

(c) The Royal College of Psychiatrists 2018.
Marco Colizzi (pictured) is a psychiatrist and clinical researcher interested in psychosis and addiction. He is studying the neurocognitive and neurochemical effects of cannabinoids on the human brain. Robin Murray is a professor of psychiatric research and has spent much of his life studying the causes of psychosis.
Cannabis is used by approximately 200 million people across the world. The current trend to popularise its medicinal properties, real and imagined, ${ }^{1}$ and to decriminalise or legalise it in many countries, is likely to be followed by greater use. ${ }^{2,3}$ However, cannabis is not as safe as was once thought. ${ }^{1,4}$ Just as longitudinal studies of tobacco smokers versus non-smokers nailed the link between cigarettes and lung cancer, so similar prospective studies have shown that heavy cannabis use carries with it an increased risk of psychosis.

Of 13 longitudinal studies in the general population, 10 have shown that cannabis users are at significant increased risk of subsequently developing psychotic symptoms or schizophrenia-like psychotic illness. The remaining three studies showed a trend in the same direction; two had a short follow-up period and the third limited power. ${ }^{4}$ A recent meta-analysis ${ }^{5}$ reported that the odds ratio for developing psychotic symptoms or a psychotic disorder in individuals who had used cannabis over non-users reached 3.9 (95\% CI 2.84-5.34) among the heaviest users.

Of course, association does not prove causation. However, one by one, alternative explanations have gradually been disproved. ${ }^{4}$ Patients do not start using cannabis to self-medicate their psychotic or prodromal symptoms or side-effects of drugs, but rather use it for the same reasons as the rest of the population, principally for its 'high'. The risk of psychosis remains after controlling for personality disorder and use of other psychotogenic drugs. Some overlap between genes carrying susceptibility to schizophrenia and to drug use has been reported but insufficient to explain more than a fraction of the relationship. ${ }^{4}$

Of course, the vast majority of people using cannabis do not develop a psychotic disorder. Not surprisingly, people with a paranoid or 'psychosis-prone' personality are especially vulnerable, alongside people with other risk factors for psychosis such as childhood trauma. Starting use in adolescence and having a family history of psychosis also carry more risk; some evidence points to variants of genes involved in the dopamine system conveying susceptibility. ${ }^{6}$

Neuroimaging studies have begun to clarify the neural underpinning of the psychotic symptoms induced by cannabinoids. ${ }^{4}$ Unlike other drugs of misuse that have their maximum impact on dopamine in the ventral striatum, long-term cannabis use induces alterations in dopamine in the associative striatum, which recent evidence pinpoints as the locus of abnormality underlying positive psychotic symptoms.

\section{The changing nature of recreational cannabinoids}

Extract of Cannabis sativa contains over 80 different cannabinoids, with delta-9-tetrahydrocannabinol $(\Delta 9-\mathrm{THC})$ and cannabidiol (CBD) the most important. $\triangle 9$-THC is the main psychoactive ingredient, and administering it experimentally to healthy volunteers can induce transient psychotic symptoms. ${ }^{4,7} \mathrm{CBD}$ appears to counteract $\Delta$ 9-THC-induced psychotic symptoms and cognitive impairment, and may even have antipsychotic properties. ${ }^{7}$

Most traditional forms of cannabis such as marijuana or hashish used in the 1960s and 1970s contained less than 4\% of THC and often an equal proportion of the ameliorating CBD. However, these have been displaced by stronger varieties in many countries. ${ }^{4}$ In the UK, the type colloquially known as skunk now dominates the market; it contains on average $16 \%$ THC; CBD is barely detectable as the plant cannot produce high concentrations of both cannabinoids. In Holland forms of Nederwiet containing up to $60 \%$ THC can be lawfully smoked in coffee shops. In Colorado, where cannabis has been legalised for recreational use, preparations such as wax dabs containing up to $90 \%$ THC can be bought.

In the last 5 years, synthetic cannabinoids, often termed collectively spice, have hit the market. In contrast to THC which is a partial agonist at the cannabinoid $\mathrm{CB}_{1}$ receptor, most synthetic cannabinoids are full agonists and consequently more powerful. Acute anxiety and paranoid reactions are common but because new molecules are constantly being produced and few have been tested in animals, incidental toxic reactions can be dangerous. ${ }^{8}$ Difficulty in detecting synthetic cannabinoids in urine has made them especially popular in prisons.

\section{Need for much more research}

Little effort has been put into studying cannabis, compared with that into alcohol or other recreational drugs. Effects of cannabis use on other psychiatric disorders need to be further examined, with some early reports claiming its use is beneficial for disorders such as posttraumatic stress disorder and depression and others that it increases their risk. ${ }^{1}$ The role of cannabis composition needs to be further examined in such studies, as it is still unclear whether at specific concentrations CBD might outweigh any harmful effects of $\Delta 9$ THC. $^{7}$ The role of cannabis dependence in perpetuating use 
deserves more study as does the possible synergistic effects of tobacco and cannabis, a major issue as the two are commonly smoked together.

Research into the numerous components of cannabis should be encouraged since, like research into opiates, it may produce drugs with important medical uses. Individual cannabinoid components can then be subject to trials measuring their effectiveness for a variety of ailments (for example pain, childhood epilepsy) in the same way any other proposed drug is evaluated. When effective, it should be introduced for prescription by doctors; several cannabinoid drugs already have become available in this manner.

\section{What should we do now?}

We psychiatrists need to be more alert to cannabis use by our patients and take as detailed a history of drug use as we do of alcohol consumption. Certain characteristics should give rise to particular suspicion. ${ }^{4}$ Patients who develop psychosis following misuse of cannabis tend to have an earlier onset of illness and to have better premorbid cognition and social function than other patients with schizophrenia. We need to get better at detecting those patients with established psychosis who continue to use cannabis, especially high potency varieties, as this is associated with worse outcomes; ${ }^{9}$ continued cannabis use and poor adherence to antipsychotics tend to go together. ${ }^{10}$

There is no strong evidence that any particular psychological intervention is particularly helpful in aiding patients to stop using cannabis. Nor have there been formal studies of which antipsychotic is best although some evidence suggests that clozapine is less likely to increase craving.

A curious divide has opened up between North America and the UK. In the USA, cannabis use in young people has increased since the mid-1990s as the number regarding use of cannabis as risky has fallen; use and potency of the drug is greater in those states that have legalised cannabis for medicinal or recreational purposes. ${ }^{2}$ In contrast, use has fallen in England; in 1996, 25.8\% of people aged 1624 admitted to having used cannabis in the previous year; by 2016, that number had declined to $16.4 \%$.

This decline has occurred in spite of the fact that use of cannabis has, in practice, been decriminalised in most parts of the UK. But, should it be legalised? This was the policy of the Liberal Democrats at the last election - although it did not turn out to be a vote winner. Indeed, it is the case both in the USA and the UK that much of the pressure to legalise is not coming from the public but rather from investors keen to make a fast buck. Would legalisation in the UK lead to an increase in consumption and cannabis tourism as evident in Amsterdam and Colorado, or could it be combined with education so that consumption would actually fall? The honest answer is that no one knows.

The sensible thing is to watch what happens in the next few years as different models of legalisation are implemented in different states in North America. The USA and Canada have embarked on a major pharmaceutical experiment with the brains of their youth, and we should wait and see the outcome of the experiment. While we wait, we need public education to make the public aware of the risks associated with heavy cannabis use. It would be a shame when we are in sight of ridding the country of the scourge of tobacco use, if it were to be replaced by use of a drug that, although less harmful to the body, is more toxic to the mind.

Marco Colizzi, MD, National Institute for Health Research (NIHR) Biomedical Research Centre (BRC), South London and Maudsley NHS Foundation Trust, UK; Department of Psychosis Studies, Institute of Psychiatry, Psychology and Neuroscience, King's College London, UK; Robin Murray, FRS, FRCPsych, National Institute for Health Research (NIHR) Biomedical Research Centre (BRC), South London and Maudsley NHS Foundation Trust: Department of Psychosis Studies, Institute of Psychiatry, Psychology and Neuroscience, King's College London, UK; Department of Psychiatry, Experimental Biomedicine and Clinical Neuroscience (BIONEC), University of Palermo, Italy

Correspondence: Marco Colizzi, Department of Psychosis Studies, Institute of Psychiatry, Psychology and Neuroscience, King's College London, London SE5 8AF, UK. Email: marco.v.colizzi@kcl.ac.uk

First received 12 Sep 2017, accepted 13 Dec 2017

\section{Acknowledgements}

We thank Professor Wayne Hall for his comments on a draft.

\section{References}

1 National Academies of Sciences, Engineering, and Medicine. The Health Effects of Cannabis and Cannabinoids: The Current State of Evidence and Recommendations for Research. The National Academies Press, 2017.

2 Hasin DS, Sarvet AL, Cerdá M, Keyes KM, Stohl M, Galea S, et al. US adult illicit cannabis use, cannabis use disorder, and medical marijuana laws: 1991-1992 to 2012-2013. JAMA Psychiatry 2017; 74: 579-88.

3 Hall W, Lynskey M. Evaluating the public health impacts of legalizing recreational cannabis use in the United States. Addiction 2016; 111: 1764-73.

4 Murray RM, Englund A, Abi-Dargham A, Lewis DA, Di Forti M, Davies C, et al. Cannabis-associated psychosis: neural substrate and clinical impact. Neuropharmacology 2017; 124: 89-104.

5 Marconi A, Di Forti M, Lewis CM, Murray RM, Vassos E. Meta-analysis of the association between the level of cannabis use and risk of psychosis. Schizophr Bull 2016; 42: 1262-9.

6 Colizzi M, Iyegbe C, Powell J, Blasi G, Bertolino A, Murray RM, et al. Interaction between DRD2 and AKT1 genetic variations on risk of psychosis in cannabis users: a case-control study. NPJ Schizophr 2015; 1: 15025.

7 Englund A, Freeman TP, Murray RM, McGuire P. Can we make cannabis safer? Lancet Psychiatry 2017; 4: 643-8.

8 Altintas M, Inanc L, Oruc GA, Arpacioglu S, Gulec H. Clinical characteristics of synthetic cannabinoid-induced psychosis in relation to schizophrenia: a singlecenter cross-sectional analysis of concurrently hospitalized patients. Neuropsychiatr Dis Treat 2016; 12: 1893-900.

9 Schoeler T, Petros N, Di Forti M, Klamerus E, Foglia E, Ajnakina O, et al. Effects of continuation, frequency, and type of cannabis use on relapse in the first 2 years after onset of psychosis: an observational study. Lancet Psychiatry 2016; 3: 947-53.

10 Colizzi M, Carra E, Fraietta S, Lally J, Quattrone D, Bonaccorso S, et al. Substance use, medication adherence and outcome one year following a first episode of psychosis. Schizophr Res 2016; 170: 311-7. 